\title{
MECHANICAL CONSTRAINT ARRANGEMENT AND ITS MULTIBOND GRAPH REPRESENTATION
}

\author{
IVAN I. KOSENKO* \\ *Institute of Applied Mathematics and Physics \\ Moscow Aviation Institute (National Research University) \\ Volokolamskoe shosse 4, 125993, Moscow, Russia \\ e-mail: kosenkoii@gmail.com
}

Key words: Multibond Graph, Wrenches, Twists, Representation of Constraint, ObjectOriented Modeling, Junction Structure

\begin{abstract}
When developing a computer model of the multibody system (MBS) dynamics it is interesting to have a unified technology to construct the models in an efficient way. It turns out object-oriented approach provides a tools to resolve such a problem successively step by step. One of these unified ways is connected tightly with the so-called multiport representation of the models initially based on the bond graph use. These latter ones in turn based on the idea of energy exchanges, and substantially on energy conservation for physically interconnected subsystems of any engineering type.

A detailed description of the multibond graph representation for the most general type of constraint is presented. It turned out the resulting total multibond graph model of the multibody system dynamics always has exactly a canonical junction structure. This representation has a tight correspondence with our previous object-oriented implementation of the mechanical constraint architecture.

Computational experience makes it possible to classify objects of the multibody system dynamics. Such a model includes two classes of objects. They correspond to notions of "body" and "constraint". Each of these notions indeed corresponds to the certain type of the multibond graph junction.
\end{abstract}

\section{INTRODUCTION}

When developing a computer model of the MBS it is interesting to have a unified technology to construct the models in an efficient way [1]. It turns out Modelica language provides a tools to resolve such a problem successively step by step using its natural approaches $[2,3]$. One of them is connected tightly with the so-called multiport representation of the models initially based on the bond graph use [4]. These latter in turn based on the idea of energy interaction [5], and substantially on energy conservation for physically interconnected subsystems of any engineering type. 
A lot of methods to describe the structure of the MBS using different graph approaches is known, see for instance [6]. Consider the MBS consisting of $m+1$ bodies $B_{0}, \ldots, B_{m}$. Represent it as a set $\mathcal{B}=\left\{B_{0}, \ldots, B_{m}\right\}$. Here $B_{0}$ is assumed to be a base body. We suppose $B_{0}$ to be connected with an inertial frame of reference, or to have a known motion with respect to the inertial frame of reference. For example one can imagine the base body as a rotating platform, or as a vehicle performing its motion according to a given law. For definiteness and simplicity we suppose in the sequel all state variables describing the rigid bodies motion always refer to one fixed inertial coordinate system connected to the base body by default.

Some bodies are considered as connected by mechanical constraints. Suppose all constraints compose the set $\mathcal{C}=\left\{C_{1}, \ldots, C_{n}\right\}$. We include in our considerations constraints of the following types: holonomic/nonholonomic, scleronomic/rheonomic.

Thus one can uniquely represent a structure of the MBS via a undirected graph $G=$ $(\mathcal{B}, \mathcal{C}, \mathcal{I})$. Here $\mathcal{I} \subset \mathcal{C} \times \mathcal{B}$ is an incidence relation setting in a correspondence the vertex incident to every edge $C_{i} \in \mathcal{C}$ of the graph. According to physical reasons it is easy to see that for any mechanical constraint $C_{i}$ there exist exactly two bodies $B_{k}, B_{l} \in \mathcal{B}$ connected by this constraint.

2 CONSTRAINT REPRESENTATION VIA BOND GRAPHS

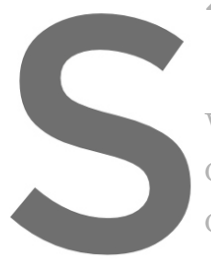

Previously, when cont

way, any physical inter

classes of the kinemats

connectors. It turned

model of the bodies 1100
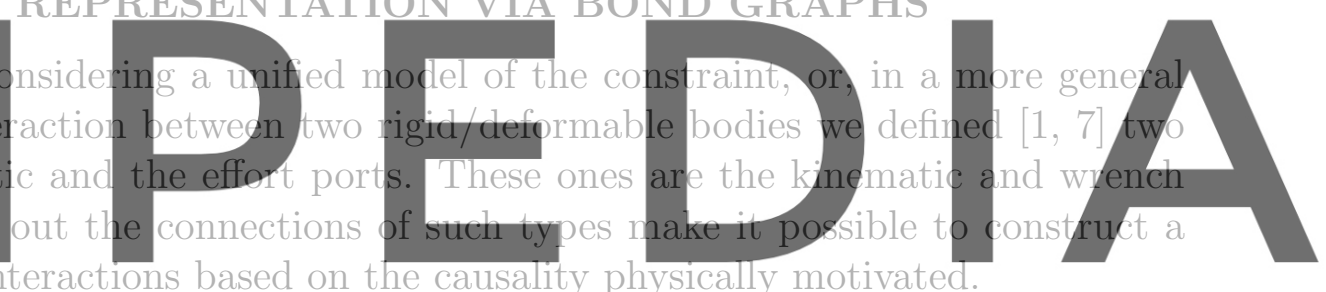

Register for free at. https/.www.scipedia.com to downlowad the version without the watermark objects of interadting bodies and reciprocally exports it in the opposite direction. 1 hus the constraint "computes" an efforts the bodies interact by.

On the other hand geometric formalisms to represent the MBS dynamics are known [8, 15] which operates with the similar information objects: twists and wrenches. In our approach twist is defined by the KinematicPort class, and wrench obviously corresponds to our WrenchPort class. The representation under consideration is tightly connected with the power based approach to modeling, so-called bond graphs [9].

Indeed, let the rigid body kinematics be defined by the twist $(\mathbf{v}, \boldsymbol{\omega})$, where $\mathbf{v}$ is the mass center velocity, and $\boldsymbol{\omega}$ is the body angular velocity. Further let all the forces acting upon the body be reduced to the wrench $(\mathbf{F}, \mathbf{M})$ with the total force $\mathbf{F}$ and the total torque $\mathbf{M}$. Thus the total power of all the forces acting on the body is computed by the known formula

$$
W=(\mathbf{v}, \mathbf{F})+(\boldsymbol{\omega}, \mathbf{M})
$$

using to represent a multibond in the bond graphs simulating the MBS dynamics. We have in such the case an evident canonical duality between twists and wrenches. 


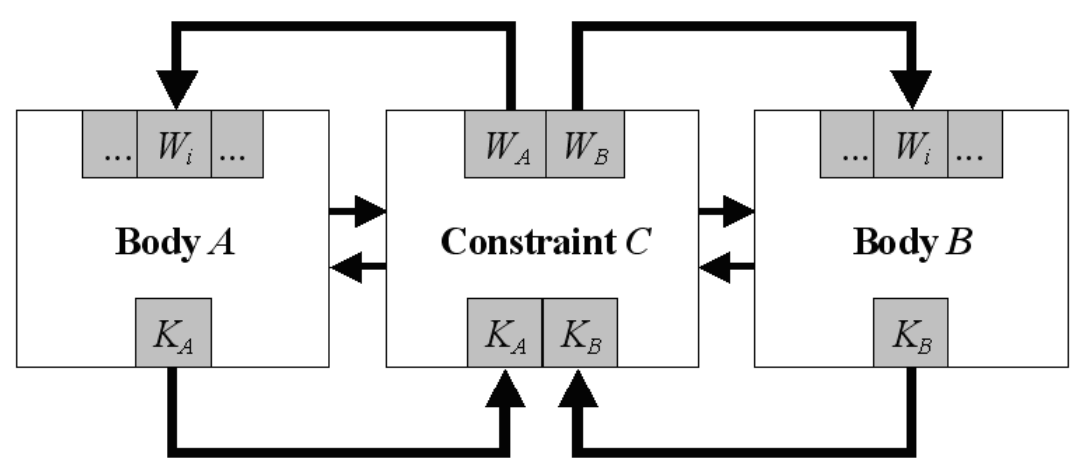

Figure 1: Architecture of Constraint

Sometimes wrenches are selected as flow variables. In other cases twists play this role. For instance similarities between electricity and mechanics cause the parallelism for electric current and forces/torques in one dimensional powertrains of mechanisms. In this ease we can set a correspondence between the Kirchhoff law for currents and the d'Alembert principle for external forces and forces of inertia "acting" upon the body.

In our opinion it may be interesting enough to apply an approach dual to the first one mentioned above.

and assumes twist for an illustration for thin chanical constraints interpreted in both Let us trace now
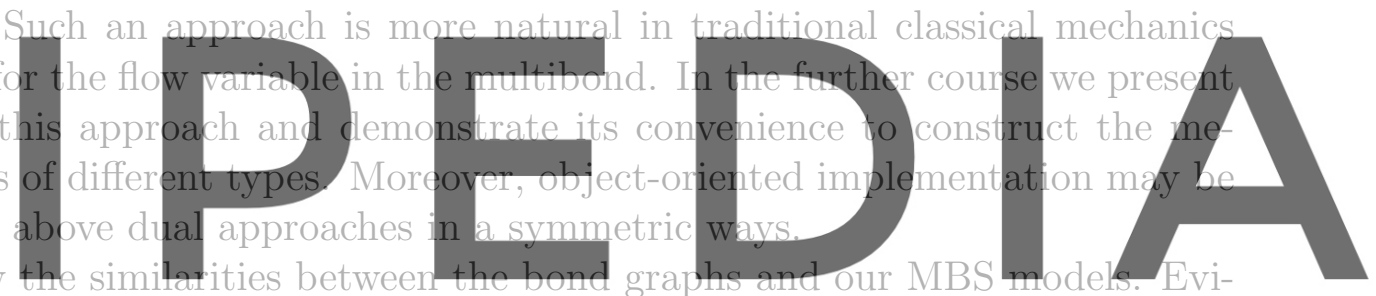

dently the pair of classes KinematicPort/WrenchPort plays a role of the multiport notion,

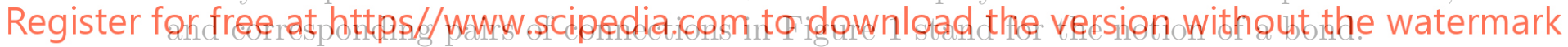

Furthermore, in this way we can associate an object of the RigidBody class with 1junction, while 0-junction is associated with the object of the class Constraint. The relevant general bond graph representation of the constraint in any MBS may be depicted as it shown in Figure 2.

All multibonds here consist of the twist $(\mathbf{v}, \boldsymbol{\omega})$ signals representing the flow component, and the wrench $(\mathbf{F}, \mathbf{M})$ signals as an effort. Causality of an inertance elements arranges according to the Newton-Euler system of ODEs. Left and right transformers are to shift the twist from the mass center to the contact point according to the known Euler formula: $(\mathbf{v}, \boldsymbol{\omega}) \mapsto(\mathbf{v}+[\boldsymbol{\omega}, \mathbf{r}], \boldsymbol{\omega})$, where the vector $\mathbf{r}$ begins at the corresponding center of mass and ends at the contact point. Reciprocally the wrenches shift to the body mass center from point of the contact in a following way: $(\mathbf{F}, \mathbf{M}) \mapsto(\mathbf{F}, \mathbf{M}+[\mathbf{r}, \mathbf{F}])$. As one can see easily the transformers conserve the power.

Central transformer is responsible for the transfer to orthonormal base at the contact point with the common normal unit vector and two others being tangent ones to both contacting bodies' surfaces supposed regular enough. For definity we interpret here the 


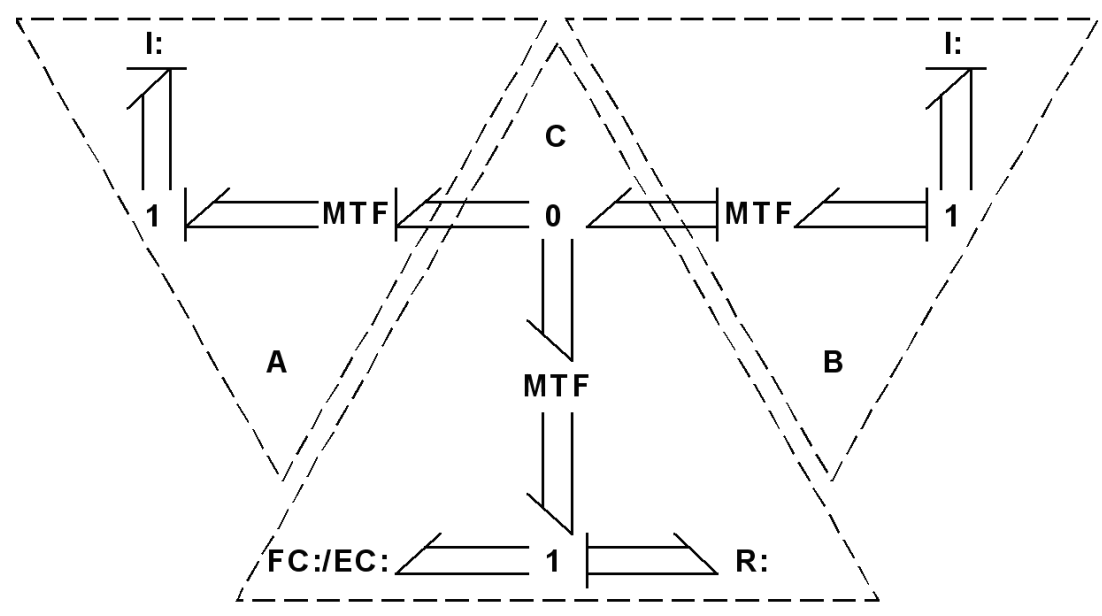

Figure 2: Architecture of Constraint: Bond Graph Representation

case of usual contact interconnection between the bodies by their outer/inner surfaces. If the inertial coordinates of these vectors compose columns of the orthogonal rotational matrix $Q$ then shifting from bottom to top across the transformer in Figure 2 we will have for the flow signals: $(\mathrm{v}, \omega) \mapsto\left(Q_{\mathrm{v}}, Q \boldsymbol{\omega}\right)$. Likewise when shifting in a reverse direction we

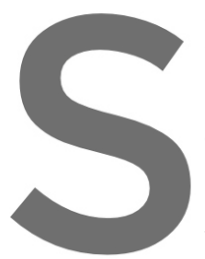
have a transformation Organization of the exactly the relative Note that it is a ticular because of it
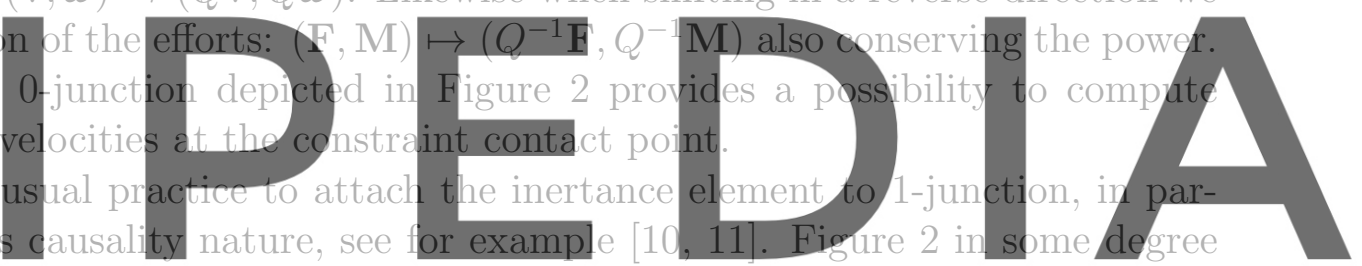

can remind us an element of the lumped model for the flexible beam dynamics.


each particular scalar bond 12] depending on the type of the constraint and is assigned finally after the whole MBS model compilation. For instance, if the constraint is of the slipping type at a contact then supposing decompositions of the relative velocities and contact forces $\mathbf{v}=\mathbf{v}_{n}+\mathbf{v}_{\tau}, \mathbf{F}=\mathbf{F}_{n}+\mathbf{F}_{\tau}$ we have the following flow constraint, element $\mathrm{FC}$, $\mathbf{v}_{n}=\mathbf{0}$ representing one scalar kinematic equation for the normal relative velocity, and the effort constraint, element EC, $\mathbf{F}_{\tau}=\mathbf{0}, \mathbf{M}=\mathbf{0}$ representing two scalar equations for the tangent contact force plus three scalar equations for the contact torque. Nonzero tangent force at the contact may arise due to the resistive element, see the bottom right multibond. If we will continue to build the bond graph model for the whole MBS in a proposed way then finally we can arrive exactly to the so-called canonical junction structure [12] useful for the formal procedures of the bond graph optimal causality assignment. For this we have to add an intermediate 0 -junctions for elements attached to 1 -junction in the constraint component $C$, see Figure 2.

Leaving some multibonds without the causality assignment and trusting this work to compiler we apply a so-called acausal modeling $[13,14]$. On the other hand if we will 




Figure 3: Bond Graph of Constraint with Compliance

act in a manner close to the real cases of constraints with the flexibility then instead of the constraint elements FC/EC, we have to use an element of the compliance with the causality uniquely determined, see Figure 3.

Further we analyze one example of the constraint frequently occurring in engineering applications: we consider an object chassification of the jo

unilateral constraints [7] doesn't change anything in principle. The only difference is that

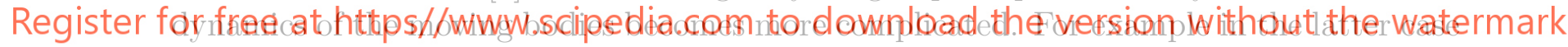

a vehicle under simulation gets an ability to bounce over the uneven surface it rolls on. In addition, its wheels can slip while moving. Thus in frame of the current paper we suppose that nonholonomic constraints implemented exactly, without any slip or separation with respect to (w. r. t.) the surface.

Remind that according to our technology of the constraint construction [1] two connected bodies are identified by convention with the letters $A$ and $B$ fixed for each body. All kinematic and dynamic variables and parameters concerned one of the bodies are equipped with the corresponding letter as a subscript.

Class Joint plays a key role in the future model of a vehicle we will build. Joint is a model derived from the base class Constraint. Remind [7] that in order to make a complete definition of the constraint object behavior for the case of rigid bodies one has to compose a system of twelve algebraic equations w. r. t. to twelve coordinates of vectors $\mathbf{F}_{A}, \mathbf{M}_{A}, \mathbf{F}_{B}, \mathbf{M}_{B}$ constituting the wrenches acting upon the connected bodies.

First six equations always present in the base model Constraint due to Newton's third law. For definity suppose these six equations are used to express six components of $\mathbf{F}_{B}$, 
$\mathbf{M}_{B}$ depending on $\mathbf{F}_{A}, \mathbf{M}_{A}$. Thus six components of $\mathbf{F}_{A}, \mathbf{M}_{A}$ remain as unknowns. To determine them each constraint of rigid bodies need in six additional independent algebraic equations. These equations can include components of force and torque directly, or be derived from the kinematic relations corresponding to specific type of the constraint.

In the case of the joint constraint being investigated here let us represent the motion of the body $B$ as a compound one consisting of the body $A$ transporting motion w. r. t. an inertial frame of reference which is similar to the Modelica Standard MultiBody Library model World, and a relative motion w. r. t. the body $A$. An absolute motion is one of the body $B$ w. r. t. inertial system.

Define the joint constraint with help of the following parameters: (a) a unit vector $\mathbf{n}_{A}$ defining in the body $A$ an axis of the joint; (b) a vector $\mathbf{r}_{A}$ fixed in the body $A$ and defining a point which constantly stays on the axis of the joint; (c) a vector $\mathbf{r}_{B}$ fixed in the body $B$ and defining a point which also constantly stays on the axis of the joint. The main task of the base joint class is to keep always in coincidence the geometric axes fixed in each of the bodies.

First of all one has to compute the radii vectors of the points fixed in the bodies w. r. t. inertial system

$$
\mathbf{R}_{\alpha}=\mathrm{r}_{O_{\alpha}}+T_{\alpha} \mathrm{r}_{\alpha} \quad(\alpha=A, B)
$$


marked point of the body $B$ defined by the position $\mathbb{R}_{B}$ we have


Register for free at https//www.scipedia.com to download the version without the watermark

$$
\begin{aligned}
\mathbf{v}_{B a} & =\mathbf{v}_{B e}+\mathbf{v}_{B r}, \\
\mathbf{v}_{B a} & =\mathbf{v}_{O_{B}}+\left[\boldsymbol{\omega}_{B}, T_{B} \mathbf{r}_{B}\right], \\
\mathbf{v}_{B e} & =\mathbf{v}_{O_{A}}+\left[\boldsymbol{\omega}_{A}, \mathbf{R}_{B}-\mathbf{r}_{O_{A}}\right],
\end{aligned}
$$

where $\mathbf{v}_{B a}, \mathbf{v}_{B e}, \mathbf{v}_{B r}$ are an absolute, transporting, and relative velocities of the body $B$ marked point, $\boldsymbol{\omega}_{A}, \boldsymbol{\omega}_{B}$ are the bodies angular velocities.

Furthermore, according to the computational experience of the dynamical problems simulation the precompiler work is more regular if the kinematic equations are expressed directly through accelerations. Indeed, otherwise the compiler tries to perform the formal differentiation of equations for the velocities when reducing an index of the total DAE system. Frequently this leads to the problems either in time of translation or when running the model.

In the first case usually diagnostics of the compiler essentially helps the developer. In the second case the model has an unpredictable behavior, and only manual preliminary reduction "regularizes" the simulation process. Thus we differentiate equations (1) and 
obtain an equations for the relative linear acceleration in the form

$$
\begin{aligned}
& \mathbf{a}_{B a}=\mathbf{a}_{O_{B}}+\left[\boldsymbol{\epsilon}_{B}, T_{B} \mathbf{r}_{B}\right]+\left[\boldsymbol{\omega}_{B},\left[\boldsymbol{\omega}_{B}, T_{B} \mathbf{r}_{B}\right]\right], \\
& \mathbf{a}_{B e}=\mathbf{a}_{O_{A}}+\left[\boldsymbol{\epsilon}_{A}, \mathbf{R}_{B}-\mathbf{r}_{O_{A}}\right]+\left[\boldsymbol{\omega}_{A},\left[\boldsymbol{\omega}_{A}, \mathbf{R}_{B}-\mathbf{r}_{O_{A}}\right]\right], \\
& \mathbf{a}_{B a}=\mathbf{a}_{B e}+2\left[\boldsymbol{\omega}_{A}, \mathbf{v}_{B r}\right]+\mathbf{a}_{B r}, \\
& \mathbf{a}_{B r}=\mu \mathbf{n}_{A i},
\end{aligned}
$$

where $\mathbf{a}_{B a}, \mathbf{a}_{B e}, \mathbf{a}_{B r}$ are an absolute, transporting, and relative accelerations of the body $B$ marked point, $\boldsymbol{\epsilon}_{A}, \boldsymbol{\epsilon}_{B}$ are the bodies angular accelerations.

We also need in an analytic representation of the conditions that the only projections of the bodies angular velocities and accelerations having a differences are ones onto the joint axis. Corresponding equations have a form

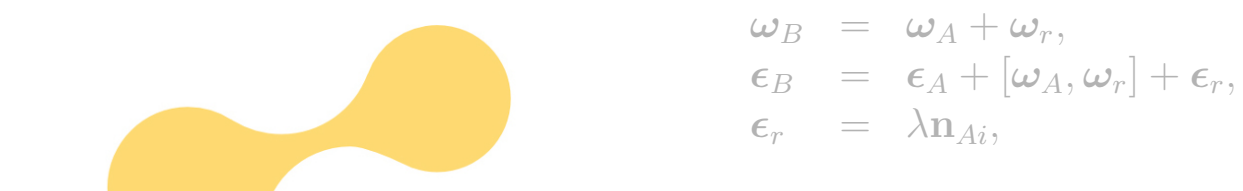

where $\boldsymbol{\omega}_{r}, \boldsymbol{\epsilon}_{r}$ are the relative angular velocities and accelerations.

Besides the kinematic scalars $\mu, \lambda$ we will need in their reciprocal values $F=\left(\mathbb{F}_{A}, \mathbf{n}_{A i}\right)$,

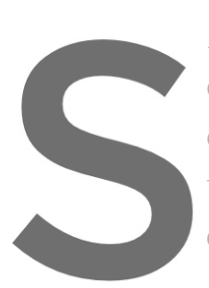
$M=\left(\mathrm{M}_{A}, \mathrm{n}_{A i}\right) \operatorname{corr}$

can be used to produce complete description two equations. One equation is intended

Regarding the gern
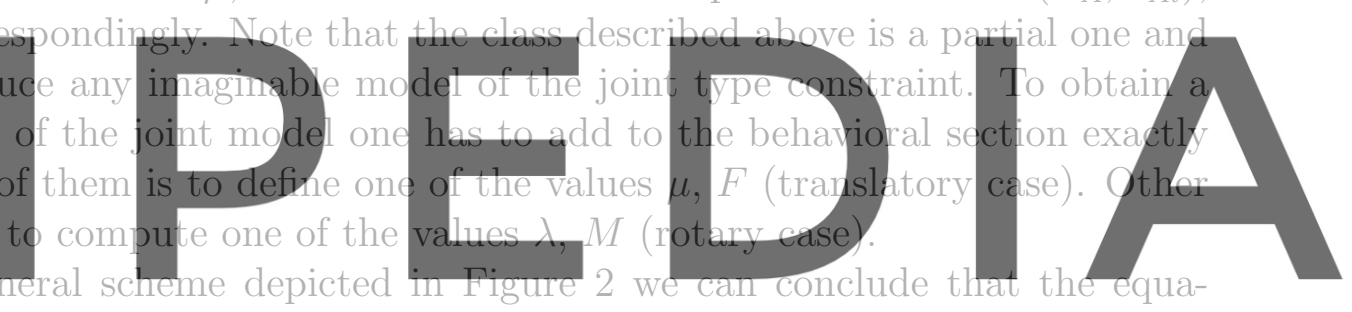

tions (1), (2), (3) together implement implicitly the constraint transformer to the joint

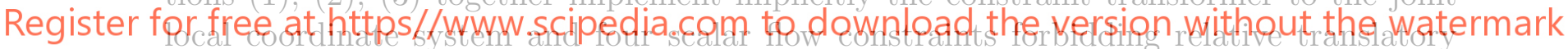

and rotary motions in the direction orthogonal to the joint axis. For derived classes only

two free scalar bonds remain.

Here we encounter the known complementarity rules once more in a way similar to one described in [7]. In our context the variables in the pairs $(\mu, F),(\lambda, M)$ are mutually complement, where one of $\mu, \lambda$ is to be utilized for the flow constraint and one of $F$, $M$ is used to compose the effort constraint. All the variables mentioned complete the set of constraints for the remaining yet unused joint axis creating thus two final scalar constraint elements in the bond graph of Figure 2.

Namely, the equations (2) implementing the Coriolis theorem for accelerations simultaneously implement, in an implicit manner, two scalar flow constraints, FC-elements, from the bottom left corner of the multi-bondgraph model in Figure 2. These flow constraints due to compiler restrictions constructed using accelerations instead of the velocities being used in a classic bond graph approach. The constraints have an obvious kinematic sense: they prevent the relative motion of the body $B$ marked point in two directions normal to the joint axis fixed in the body $A$. 
In addition, the equations (3) implement two other scalar flow constraints, this time for the rotary motion. These constraints forbid the relative rotation of the body $B \mathrm{w}$. r. t. body $A$ about two axes each normal to the joint axis mentioned above which is rigidly connected with the body $A$.

Note, that the construct of equations (2) and (3) is such that they allow the body $B$ relative motion along and about the joint axis of the body $A$ thus implementing the kinematic pair with two DOFs. Returning to Figure 2 of the general constraint multibond graph we can conclude that the vertical multibond attached to 0 -junction implements flow variables corresponding to the relative body $B$ motion w. r. t. body $A$ in inertial coordinates. Such a description supposes an existence of the special coordinates reference frame connected with the body $A$ at its joint constraint marked point. The transformation to these coordinates is implemented exactly via corresponding transformer, central in the triangle block $C$. The transformer itself nests in formulae of equations (2) and (3).

Consider several examples of the classes derived from the Joint model for the several particular types of joints. The model FixedIdealJoint is defined by the equations

$$
\mu=0, \quad M=0
$$

and prevents the relative motion along the joint axis but allows free rotation about it.

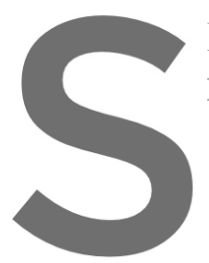

It is exactly a revolu

FreeIdeal Joint is defined br


permitting free transtation along and free rotation about
Class SpringIdeal Joint described by the equations

Register for free at https//WwW.scipedia.com to download the version without the watermark

$$
F=c \nu+d \dot{\nu}, \quad M=0, \quad \ddot{\nu}=\mu
$$

with an initial data $\nu\left(t_{0}\right)=0, \dot{\nu}\left(t_{0}\right)=0$ for the relative translatory position $\nu$ provides a viscoelastic compliance with the stiffness $c$ and damping $d$. The rotary motion remains free. This model is useful to simulate almost rigid constraints to avoid the potential problems with so-called statically undefinable systems of forces acting upon the ideal rigid bodies.

The model FixedControlledJoint with the behavior defined by the equations

$$
\mu=0, \quad M=f(t, \varphi, \dot{\varphi}), \quad \ddot{\varphi}=\lambda
$$

provides the rotating torque as a control effort with the prescribed control function $f(t, \varphi, \dot{\varphi})$. Initial data $\varphi\left(t_{0}\right)=\varphi_{0}, \dot{\varphi}\left(t_{0}\right)=\dot{\varphi}_{0}$ are prepared according to the initial data concerning the joint. From the bond graph viewpoint the second equation in (4) can be implemented as a combination of the source effort, compliance, and resistance elements. This type of joint corresponds to the Revolute joint constraint of Modelica 
Standard Library from the ModelicaAdditions package. Such a joint can be driven by the electromotor.

The model FreeSlideJoint defined by the equations

$$
F=0, \quad \lambda=0
$$

provides free, without any resistance, relative sliding along the joint axis without any rotation about it. As one can see this is a prismatic type of joint.

We can reformulate the FixedControlledJoint model creating the model FixedServoJoint in a following useful way

$$
\mu=0, \quad \lambda=f(t, \varphi, \dot{\varphi}), \quad \ddot{\varphi}=\lambda
$$

thus composing a kinematic restricting constraint, so-called servoconstraint. The function $f(t, \varphi, \dot{\varphi})$ supposed as a prescribed one. Initial data for the angle $\varphi$ of the relative rotation are prepared in the same way as for (4). It is clear one can create a lot of other different combinations of equations to construct the joint constraints needed in engineering applications.

The derived joint classes described here are to close the system of kinematic equations (2) and (3) completing them mainly by two scalar additional equations

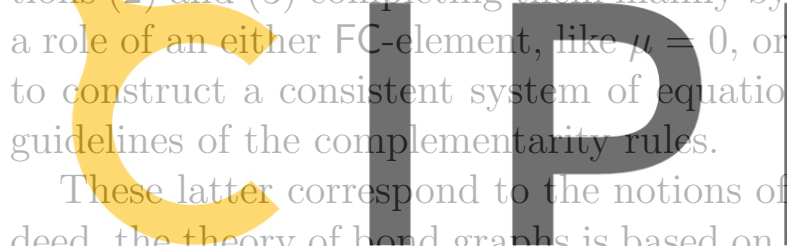
deed, the theory of bond graphs is based on
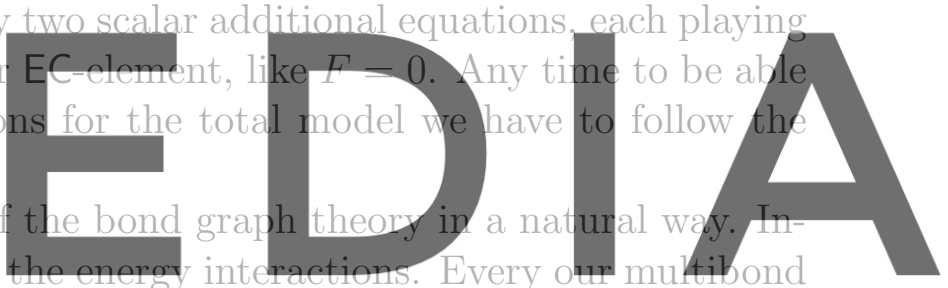

being an energy/power conductor reflects complementarity by its twist/wrench duality.

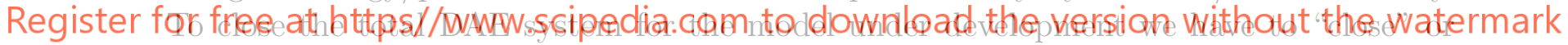
rather to "seal" each free scalar bond in EC/FC-element of the block C in Figure 2 by the corresponding one scalar equation for flow or effort variable. Thüs here we outline the main rule to compose equations for the models of constraints for MBS of any type in a consistent way when applying the object-oriented approach.

See also papers $[16,17,18]$ for particular cases of non-linear contact problems.

\section{CONCLUSIONS}

The brief list of concluding remarks is of the following one:

- A unified multibond graph representation of the MBS dynamics in a sufficiently simple way with the canonical junction structure is possible.

- The representation depicted in Figure 2 can be used as a guideline to construct the consistent system of DAEs in a systematic way. In other words we can say that multibond graph constructs like ones of Figure 2 are to be used as a regular basis for more informal object-oriented approach. 
- An acausal modeling accelerates the model development releasing a project team from the problem of causality assignment if one takes into account some requirements like complementarity rules.

- Introducing the compliance into the model may be useful and efficient preserving the principal properties of the MBS like anholonomity etc.

\section{ACKNOWLEDGEMENT}

This work has been prepared at MAI and the Lomonosov MSU.

\section{REFERENCES}

[1] Kosenko, I. I., Stepanov, S. Y. and Gerasimov, K. V. Contact tracking algorithms in case of the omni-directional wheel rolling on the horizontal surface. Multibody System Dynamics (2019) 45:273-292.

[2] Modelica - a unified object-oriented language for physical systems modeling. Tutorial. Modelica Association, (2000).



[3] Modelica - a unified object-oriented language for physical systems modeling. Lan-


[5] Karnopp D. C., The energetic structure of multibody dynamic systems. Journal of

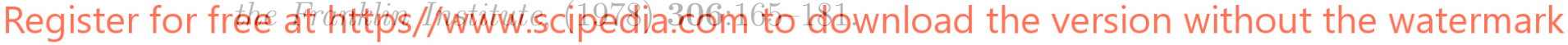

[6] Wittenburg, J. Dynamics of systems of rigid bodies. B. G. Teubner, Stuttgart, (1977).

[7] Kossenko, I. I., Implementation of Unilateral Multibody Dynamics on Modelica. Proceedings of the 4th International Modelica Conference, Hamburg University of Technology, Hamburg-Harburg, Germany, March 7-8. (2005) :13-23.

[8] Stramigioli, S., Blankenstein, G., Duindam, V., Bruyninckx, H., Melchiorri C., Power Port Concepts in Robotics. The Geometrical-Physical Approach. Tutorial at 2003 IEEE International Conference on Robotics and Automation. — IEEE, (2003).

[9] Paynter, H. M., Analysis and Design of Engineering Systems. — The M. I. T. Press: Cambridge, Massachusetts, (1961).

[10] Cellier, F. E., Continuous System Modeling. - Springer-Verlag: New York, (1991).

[11] Mukherjee, A., Karmakar, R., Modelling and Simulation of Engineering Systems through Bondgraphs. - Alpha Science International Ltd.: (2000). 
[12] Golo, G., Interconnection Structures in Port-Based Modelling: Tools for Analysis and Simulation. PhD Thesis. - University of Twente: Enschede, The Netherlands, (2002).

[13] Dymola. Dynamic Modeling Laboratory. User Manual. Volume 1. Version 7.3. Ideon Science Park, SE-223 70, Lund, Dynasim AB, (2009).

[14] Dymola. Dynamic Modeling Laboratory. User Manual. Volume 2. Version 7.3. Ideon Science Park, SE-223 70, Lund, Dynasim AB, (2009).

[15] Duindam, V., Blankenstein, G., Stramigioli S., Port-Based Modeling and Analysis of Snakeboard Locomotion. Sixteenth International Symposium on Mathematical Theory of Networks and Systems. Katholieke Universiteit Leuven, Belgium, July 5-9 (2004)

[16] Kireenkov, A. A., Modelling of the Force State of Contact of a Ball Rolling along the Boundaries of Two Rails. (2021) AIP Conference Proceedings, 2343, Paper No 120002, DOI: 10.1063/5.0047955, ISSN: 0094243X, ISBN: 9780735440883.

[17] Kireenkov, A. A., Preface: Mathematical Models and Investigations Methods of Strongly Nonlinear Systems. (2021) AIP Conference Proceedings, 2343, Paper No 120001, DOI: 10.1063/5.0047954, ISSN: 094243X, ISBN: 9780735440883.

[18] Kireenkov, A. A., Zhavoronok, S. I., Nushtaev, D. V. On Tire Models Accounting for both Deformed State and Coupled Dry Friction in a Contact Patch. (2021) Computer Research and Modeling, 13 (1), pp. 163-173. DOI: 10.20537/2076-7633-2021-13-1-163173, ISSN: 20767633. 